\title{
MATCHING THEOREMS FOR COMBINATORIAL GEOMETRIES
}

\author{
BY MARTIN AIGNER ${ }^{1}$ AND THOMAS A. DOWLING ${ }^{2}$
}

Communicated by Gian-Carlo Rota, July 18, 1969

1. Introduction. Let $G(S)$ and $G(T)$ be combinatorial geometries of finite rank on sets $S$ and $T$, respectively, and let $R \subseteq S \times T$ be a binary relation between the points of $G(S)$ and $G(T)$. By a matching from $G(S)$ into $G(T)$, we understand a one-one function $f$ from an independent set $A \subseteq S$ onto an independent set $B \subseteq T$ with $(a, f(a))$ $\in R$ for all $a \in A$. In this note, we present a characterization of matchings of maximum cardinality, a max-min theorem, and a number of related results. In the case when $G(S)$ and $G(T)$ are both free geometries, Theorems 1 and 2 reduce to "the Hungarian method" as introduced by Egerváry and Kuhn [1], and to the König-Egerváry theorem, respectively. Corollary 2 for the case when $G(S)$ is a free geometry and $G(T)$ arbitrary was first discovered by Rado [6] (see also Crapo-Rota [2]). When both $G(S)$ and $G(T)$ are free geometries, Corollary 2 reduces to the well-known SDR theorem.

2. Terminology. For an arbitrary geometry $G(S)$, the closure operator will be denoted by $J$ and the rank function by $r$. $(G(S), G(T), R)$ shall denote the system of the two geometries together with $R$, and $R\left(S^{\prime}\right)=\left\{y \mid\right.$ there is some $x \in S^{\prime}$ with $\left.(x, y) \in R\right\}$ for $S^{\prime} \subseteq S$. Let $(A, B, f)$ denote a matching from $A$ onto $B . M=\{(a, f(a)), a \in A\}$ is called the edge set of the matching $(A, B, f)$, and we adopt the convention $M=(A, B, f)$. The common cardinality of $A, B, M$ is called the size $\nu(M)$ of the matching. A support of $(G(S), G(T), R)$ is a pair $(C, D)$ of closed sets, where $C \subseteq S, D \subseteq T$, such that $(c, d) \in R$ implies at least one of $c \in C, d \in D$ holds. The order $\lambda$ of a support $(C, D)$ is defined as $\lambda(C, D)=r(C)+r(D)$. Finally, an augmenting chain with

AMS Subject Classifications. Primary 0504, 0535, 0540; Secondary 0460, 0630.

Key Words and Phrases. Combinatorial geometry, matroid, binary relation between geometries, matching, Hungarian method, augmenting chain, König-Egerváry theorem, marriage theorem, transversal pregeometry.

${ }_{1}$ Research supported by the United States Air Force Grant AFOSR-68-1406.

${ }^{2}$ Research supported in part by National Science Foundation Grant No. GU-2059. 
respect to the matching $M=(A, B, f)$ is a sequence of $2 n+1$ distinct pairs $\left(a_{0}^{\prime}, b_{1}^{\prime}\right),\left(b_{1}, a_{1}\right),\left(a_{1}^{\prime}, b_{2}^{\prime}\right), \cdots,\left(b_{n}, a_{n}\right),\left(a_{n}^{\prime}, b_{n+1}^{\prime}\right)$ such that

$$
\begin{aligned}
\left(a_{i}, b_{i}\right) & \in M, & \left(a_{i}^{\prime}, b_{i+1}^{\prime}\right) & \in R-M, \\
a_{0}^{\prime} & \in S-J(A), & b_{n+1}^{\prime} & \in T-J(B), \\
a_{i}^{\prime} & \in J(A), & a_{i}^{\prime} & \notin J\left(\left(A-\bigcup_{j=1}^{i} a_{j}\right) \cup \bigcup_{j=1}^{i-1} a_{j}^{\prime}\right), \\
b_{i}^{\prime} & \in J(B), & b_{i}^{\prime} & \notin J\left(\left(B-\bigcup_{j=1}^{i} b_{j}\right) \cup \bigcup_{j=1}^{i-1} b_{j}^{\prime}\right)
\end{aligned}
$$

for $1 \leqq i \leqq n$.

\section{The main results.}

Theorem 1. A matching $M=(A, B, f)$ in $(G(S), G(T), R)$ is of maximum size if and only if there does not exist an augmenting chain with respect to $M$.

THEOREM 2. $\max _{M \text { matching }} \nu(M)=\min _{(C, D) \text { support }} \lambda(C, D)$.

Brief outline of Proof of Theorems 1 and 2. First, it is easily seen that by means of an augmenting chain we can increase a given matching $M$, since by conditions (2) and (3) the sets

$$
\begin{aligned}
& A^{\prime}=\left(A-\bigcup_{j=1}^{n} a_{j}\right) \cup \bigcup_{j=0}^{n} a_{j}^{\prime}, \\
& B^{\prime}=\left(B-\bigcup_{j=1}^{n} b_{j}\right) \cup \bigcup_{j=1}^{n+1} b_{j}^{\prime}
\end{aligned}
$$

are independent. Further, we clearly have $\nu(M) \leqq \lambda(C, D)$ for any matching $M$ and any support $(C, D)$.

Assume now there is no augmenting chain with respect to $(A, B, f)$. Put $C_{0}=S-J(A)$, then $R\left(C_{0}\right) \subseteq J(B)$. Let $B_{1}$ be the minimal subset of $B$ such that $R\left(C_{0}\right) \subseteq J\left(B_{1}\right), A_{1}=f^{-1}\left(B_{1}\right)$ and $C_{1}=S-J\left(A-A_{1}\right)$. In general, having constructed $C_{i-1}$, we define $B_{i}$ as the minimal subset of $B$ such that $R\left(C_{i-1}\right) \cap J(B) \subseteq J\left(B_{i}\right)$, and set $A_{i}=f^{-1}\left(B_{i}\right)$ and $C_{i}$ $=S-J\left(A-A_{i}\right)$. This way we construct three monotonically increasing sequences of sets $A_{i}, B_{i}, C_{i}$ and since all the $B_{i}$ 's are contained in $B$, these sequences must terminate after a finite number of, say, $m$ steps.

The crucial part of the argument consists in showing that $R\left(C_{n}\right)$ $\subseteq J(B)$ for all $n=0, \cdots, m$. This is accomplished by disproving the opposite through construction of an augmenting chain with respect 
to $M$. Now since $R\left(C_{m}\right) \subseteq J\left(B_{m}\right)$, i.e., $R\left(S-J\left(A-A_{m}\right)\right) \subseteq J\left(B_{m}\right)$, we infer that $\left(J\left(A-A_{m}\right), J\left(B_{m}\right)\right)$ constitutes a support with order equal to the size of $M$. Thus $M$ is a matching of maximum cardinality and the equality in Theorem 2 holds.

Corollary 1. For $A \subseteq S$, define the deficiency of $A$ as $\delta_{S}(A)=r(S)$ $-r(S-A)-r(R(A))$, and let $\delta_{S}=\max _{A} \subseteq S \delta_{S}(A)$. Then

$$
\max _{M \text { matehing }} \nu(M)=\min _{(C, D) \text { support }} \lambda(C, D)=r(S)-\delta_{S} .
$$

We have

$$
\begin{aligned}
r(S)-\delta_{S} & =r(S)-\max _{\Delta \subseteq S}(r(S)-(r(S-A)-r(R(A))) \\
& =\min _{A \subseteq S}(r(S-A)+r(R(A)))=\min _{A \subseteq S}(r(A)+r(R(S-A))),
\end{aligned}
$$

and the minimum is clearly obtained by some closed set $A$. But then $(A, J(R(S-A)))$ is a support for $(G(S), G(T), R)$ and the conclusion follows.

Corollary 2 (Generalized Marriage Theorem). Given $(G(S), G(T), R)$, then $\max M$ matching $\nu(M)=r(S)$ if and only if $r(S)$ $-r(S-A) \leqq r(R(A))$ for all $A \subseteq S$.

Corollary 3. Let $(A, B, f)$ be a matching in $(G(S), G(T), R)$ and suppose it is not of maximum size, then there exists a matching $\left(A^{\prime} \cup a, B^{\prime} \cup b, f^{\prime}\right)$ such that $J\left(A^{\prime}\right)=J(A), J\left(B^{\prime}\right)=J(B)$, and $a \notin J\left(A^{\prime}\right)$, $b \notin J\left(B^{\prime}\right)$.

This follows immediately from the definition of augmenting chains, part (3).

Corollary 4 (See also [2], [3], [4]). Given $(G(S), G(T), R)$, where $G(S)$ is a free geometry. Define a new independence structure on $S$ by calling $A \subseteq S$ independent if and only if there exists a matching $(A, B, f)$ for some $B$ and $f$. This defines a pregeometry on $S$, called the transversal pregeometry with respect to $(G(S), G(T), R)$.

Corollary 3 applied to $\left(G\left(S^{\prime}\right), G(T), R \cap\left(S^{\prime} \times T\right)\right)$ for $S^{\prime} \subseteq S$ shows that every independent subset $A \subseteq S^{\prime}$ as defined above can be embedded in one of maximum (and by Corollary 1, constant) size.

It should be remarked that Corollary 4 ceases to be true for arbitrary geometries $G(S)$. The function $r^{*}$ given by the definition of independent sets in Corollary 4 and by the formula in Corollary 1 as $r^{*}\left(S^{\prime}\right)=r\left(S^{\prime}\right)-\delta_{S^{\prime}}$ for $S^{\prime} \subseteq S$ is unit-increasing, but fails to be semimodular in general. For the same reason one cannot prove Theorem 
2 along the lines suggested by Ore [5] although this approach works when $G(S)$ is a free geometry.

Acknowledgment. We would like to express our gratitude to Professor Rota who suggested this work to us during a series of lectures given at the University of North Carolina in the Spring 1969.

\section{REFERENCES}

1. C. Berge, The theory of graphs and its applications, Dunod, Paris, 1958; English transl., Methuen, London and Wiley, New York, 1962. MR 21 \#1608; MR 24 \#A2381.

2. H. H. Crapo and G. C. Rota, On the foundations of combinatorial theory: combinatorial geometries, MIT Press, Cambridge, Mass., 1970.

3. J. Edmonds and D. R. Fulkerson, Transversals and matroid partitions, J. Res. Nat. Bur. Standards 69 B (1965), 147-153. MR 32 \#5531.

4. L. Mirsky and H. Perfect, Applications of the notion of independence to problems of combinatorial analysis, J. Combinatorial Theory 2 (1967), 327-357. MR 37 \#1268.

5. O. Ore, Graphs and matching theorems, Duke Math. J. 22 (1955), 625-639. MR 17, 394.

6. R. Rado, $A$ theorem on independence relations, Quart. J. Math. Oxford Ser. 13 (1942), 83-89. MR 4, 269.

University of North Carolina, Chapel Hill, North Carolina 27514 